\title{
Usefulness of CURB-65 and Pneumonia Severity Index for Influenza A H1N1v pneumonia
}

\author{
A. Estella
}

ABSTRACT: Usefulness of CURB-65 and Pneumonia Severity Index for Influenza A H1N1v pneumonia. A. Estella.

Background. Different prognostic scales have been documented to assess the severity and indications for hospitalization and ICU admissions of community acquired pneumonia. During the past two years Influenza A H1N1v infections have been commonly attended to in emergency departments. The aim of the study was to analyse the usefulness of the application of the Pneumonia Severity Index (PSI) and CURB-65 prognostic scales in patients with primary viral pneumonia caused by influenza A H1N1v.

Methods. A retrospective study was performed at a community hospital with a 17 bed-Intensive Care Unit. Patients admitted in hospital with influenza A H1N1v pneumonia over a two year period were analysed. CURB 65 and PSI scales were applied in the emergency department and outcome and destination of admission were analysed.
Results. 24 patients were registered, 19 required ICU admission and 5 patients were admitted in medical wards. Most of the patients admitted to the intensive care unit (78.9\%) required mechanical ventilation. Mortality was $21.1 \%$. Most patients admitted to the ICU had CURB 65 scale of 1 $(60 \%), 13.3 \%$ obtained 0 and $26.7 \%$ 2. PSI scale resulted class I in a $20 \%$, class II $40 \%, 26.7 \%$ class IV and $13.3 \%$ class V. The scales CURB 65 and PSI showed no differences in scores according to the destination of admission and mortality.

Conclusions. Use of CURB-65 and PSI in the emergency department may underestimate the risk of patients with Influenza A H1N1v pneumonia. Based in our results, the ability of these scales to predict ICU admissions for Influenza A H1N1v pneumonia is questioned.

Monaldi Arch Chest Dis 2012; 77: 3-4, 118-121.

Keywords: Influenza A H1N1, Pneumonia, Severity index, CURB 65, PSI, Emergency.

Intensive Care Unit, Hospital of Jerez, Jerez de la Frontera (Cádiz), Spain.

Correspondence: Angel Estella, Intensive Care Unit, Hospital of Jerez, Carretera Cadiz s/n. CP: 11407, Jerez de la Frontera (Cádiz), Spain; e-mail: litoestella@hotmail.com

\section{Introduction}

During winter and in times of viral epidemic there is a significant increase of respiratory infections; this is a common reason for visits to emergency departments (ED). The community-acquired pneumonia (CAP) is the most severe type of presentation of respiratory infection and a common disease that is associated with high morbidity and mortality $[1,2]$.

Approximately 35\% of CAP treated in emergency departments required hospitalisation. The diagnosis of CAP in the ED is of great importance in assessing indications for hospitalisation and severity criteria, these decisions are determined by both age and comorbidity of the patient and by clinical, radiological and laboratory findings.

Different prognostic scales have been documented to assess the severity of CAP; the most commonly used are the Pneumonia Severitys Index (PSI) developed by Fine et al [3] and CURB65 scale of the British Thoracic Society [4]. The ATS / IDSA periodically review the criteria of severity and need for ICU admission for this disease [5].
The pandemic caused by influenza A H1N1 experienced during 2009 was challenging for professionals in emergency services, showed a previously unknown stage, characterised not only by the high demand for services but also by the occurrence (with rapid progression) to severe respiratory distress syndrome associated with significant mortality. Those who are at greater risk of complications include the elderly, chronically ill, those with heart disease, obesity, diabetes, immunocompromised, pregnant women and patients with asthma or chronic bronchopaties, however severe cases have been described in patients without previous symptoms [6-8].

The aim of this study is to describe the clinical features of diagnosed cases of Influenza A H1N1v infection requiring hospitalization and analyzed in patients with primary viral pneumonia microbiologically confirmed the usefulness of the application of prognostic scales PSI and CURB 65.

\section{Methods}

A retrospective study in a community hospital with 533 beds which included patients treated in the emergency department with clinical suspicion 
of influenza A H1N1v and confirmed pneumonia that required hospitalisation. The time of study was 24 months during the epidemic years 2009 and 2010.

Respiratory specimens were extracted in all patients to confirm the infection, pharyngeal aspirates and/or nasopharyngeal swabs for microbiological study by conducting chain reaction polymerase in real time reverse transcription (RTPCR). Likewise, all patients were treated empirically with oseltamivir.

The variables analysed were; age, sex, symptoms present at admission in the emergency department, radiological pattern, arterial pressure, heart and respiratory rate. Apache II score at ICU admission, mechanical ventilation and vasoactive drugs requirements, time of mechanical ventilation, ICU length of stay and mortality were recorded.

Retrospectively CURB 65 and PSI scales were assessed, and an analysis of destination of patients, to medical wards or ICU, was performed.

Pregnant patients, bacterial co-infections and patients under 18 years were excluded of final analysis. Data collected was analysed using SPSS version 18 for Windows. Qualitative variables were described with the percentage distribution of each of the categories. Quantitative variables were described as mean and standard deviation when normally distributed, with the median otherwise.

\section{Results}

During the study period, 38 patients infected with Influenza A confirmed H1N1v were admitted for hospitalisation from the emergency department, most of them, $25(65.8 \%)$ were admitted to ICU, the rest in respiratory and internal medicine ward. A total of 24 patients were diagnosed with primary viral pneumonia and were finally analysed, 19 required admission to ICU.

Clinical characteristics of patients are showed in table 1. Symptoms were mostly fever and cough. According to the destination we find differences in $\mathrm{O}_{2}$ saturation at admission, that was $96.6 \pm 2 \%$ in patients admitted in medical wards and of $87.7 \pm 5 \%$ in ICU admissions, there were no differences in laboratory parameters $\mathrm{pH}, \mathrm{Na}+$, hematocrit, glucose and urea according ICU or medical wards admissions. Severe respiratory failure was the main cause of ICU admission, mortality in patients admitted to ICU was $21.1 \%$, and the cause of death was hypoxemia due to the development to acute respiratory distress syndrome. Initially, in all the patients empirical antibiotic and antiviral treatment was commenced, de-scalation of antibiotic therapy was performed according microbiologic cultures results.

In the group of patients admitted in ICU mean APACHE II score at admission was $12,4 \pm 6$. In mechanically ventilated patients duration of this treatment was $13 \pm 8$ days and ICU length of stay was $16,1 \pm 11$ days. Mean CRP level was $20,8 \pm 16$ $\mathrm{mg} / \mathrm{dl}$ and leukocyte count was $5883 \pm 2776 / \mu \mathrm{l}$.

The scales PSI and CURB 65 were applied in all patients and do not show differences according to the destination and mortality (tables 2 and 3 ).

Mechanical ventilation requirements may be considered a clinically significant severity marker, most patients admitted to ICU required mechanical ventilation $(78.9 \%)$.

\section{Discussion}

The objective of prognostic scales application in the emergency services must be to optimise the use of health care resources. During the 2009 influenza pandemic, hospital emergency departments experienced a high demand care with clinical cases that ranged from mild infections without hospitalisation requirements to serious respiratory failure with rapid progression to respiratory distress. Triage systems are an essential tool in the organisational model of response to an increase in attendance in certain circumstances such as a pandemic.

PSI and CURB scales $65[3,4]$ have been validated by the scientific community in the management of CAP to assess its severity. Decision making in an emergency service is complex, not only because of the uncertainty inherent in the practice of medicine but also due to the need to manage admissions beds in a high demand environment for care services.

The application of the CURB 65 and PSI scales are useful in the initial evaluation of CAP in the emergency services [9], it is necessary to estimate the severity, to decide on the requirements of hospital or ICU requirements based on the estimated risk. CURB 65 and PSI are based on probability and therefore involve a measure of the risk estimate; Niederman et al propose the combined application of both scales in the evaluation of CAP [10]. Based on this premise these scores were recommended in the initial management of pneumonia caused by influenza A H1N1v [11], only few studies with a short number of patients has assessed in clinical practice the usefulness of these scales [12-14], Brandão-Neto RA et al propose the use of other scales like STSS [14].

\begin{tabular}{|c|c|c|}
\hline & $\begin{array}{l}\text { Hospitalization ward } \\
\qquad \mathrm{n}=5\end{array}$ & $\begin{array}{l}\text { ICU } \\
\mathrm{n}=19\end{array}$ \\
\hline Age, years & $51,2 \pm 13,6$ & $44,8 \pm 11,4$ \\
\hline Gender $(\mathbf{M} / \mathbf{F})$ & $0 / 5$ & $12 / 7$ \\
\hline \multicolumn{3}{|l|}{ Radiological pattern (n/\%) } \\
\hline - Unilateral infiltrate & $3(60)$ & $2(10,5)$ \\
\hline - Bilateral infiltrate & $2(40)$ & $17(89,5)$ \\
\hline Arterial bllod pressure (mmHg) & $120 / 71$ & $137 / 78$ \\
\hline Respiratory rate (breaths/minute) & 17,8 & $25,4 \pm 6,8$ \\
\hline Heart rate (beats/minute) & 81,2 & $105,2 \pm 19,8$ \\
\hline Non invasive ventilation & $0 \%$ & $20 \%$ \\
\hline Mechanical ventilation & $0 \%$ & $78,9 \%$ \\
\hline Vasoactive drugs & $0 \%$ & $40 \%$ \\
\hline Mortality & $0 \%$ & $21,1 \%$ \\
\hline
\end{tabular}


Table 2. - Relationship between CURB 65 score, destination and mortality

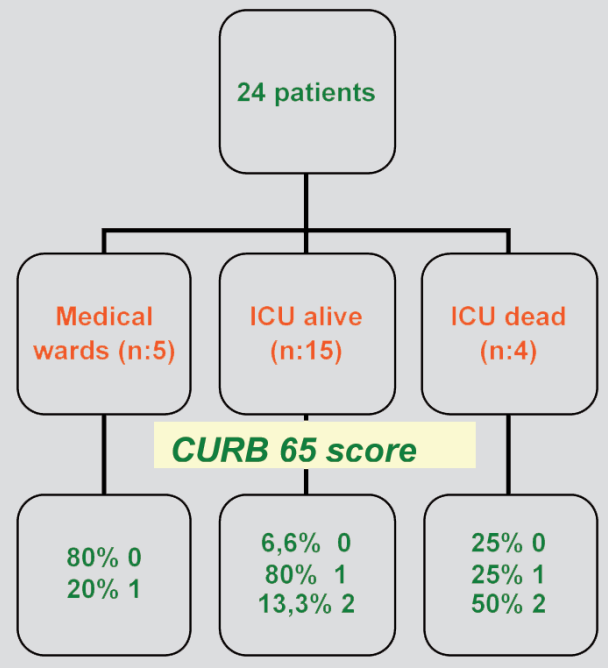

It should be noted that this infection has presented notable differences in both epidemiological and clinical and prognostic with respect to bacterial etiology of CAP, the experience gained in these two years should serve to address the disease minimising the climate of uncertainty that characterised the pandemic in its early stages. The predominant clinical profile of patients included in the study was young patients, with almost no relevant medical history. Although the application of prognostic scales most frequently used (3.4) in the management of CAP was recommended in the action protocols against influenza $\mathrm{A}$ infection H1N1v is useful in estimating the risk and need for admission or invasive measures it has not been sufficiently investigated [5-8]. In our series the application of the scales 65 and PSI CURB was not found to be useful in estimating prognosis, and thus was not helpful in making professional decisions of emergency at the time of admission and for management of destination. $80 \%$ of patients admitted to ICU had a CURB 65 score of 0 or 1 and almost two thirds had a score on the PSI classified as low risk (risk class I to III). Even into the bacterial CAP some authors have questioned the ability to predict ICU admission [12-14]. Indeed, these indices were designed and validated to predict the risk of death in patients with CAP [18], half of died patients in the study obtained a CURB 65 score of 2 and the remaining two patients had a risk score of 0 and 1 . With regard to the PSI scale were classified as I, II, III and V, highlighting the unreliability of these scales in predicting mortality for this etiology. No doubt many factors have influenced the failure as a method of estimating the prognosis of pneumonia Influenza A H1N1v, both age and comorbidity are significant factors in the scoring of these indices and the clinical profile of patients with severe pneumonia Influenza $A$ H1N1v were young with no medical history, the average age of the patients in our study was 46
Table 3. - Relationship between Pneumonia Severity Index, destination and mortality

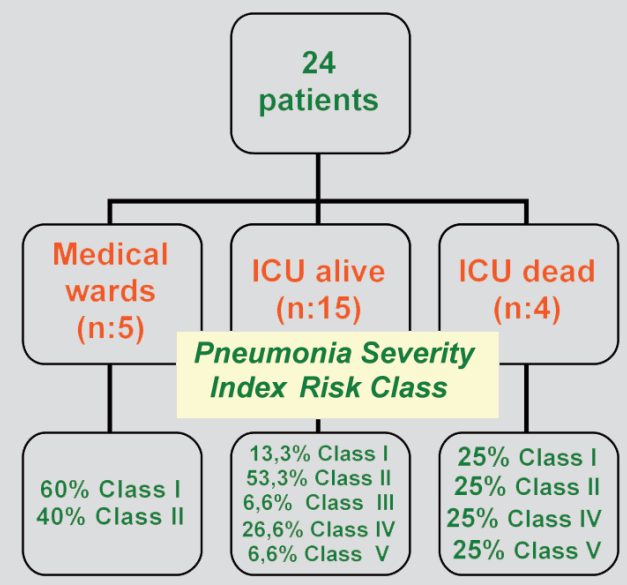

years. CURB 65 scale has the advantage over PSI is easier to perform because it has fewer variables to assess, however it does not evaluate hypoxemia, which was a constant feature in critically ill patients admitted to the ICU. Some authors defend the high negative predictive value for important adverse effects such as the need for mechanical ventilation and risk of death within 30 days [17], in our series of 15 patients who required mechanical ventilation had a low risk according to the scale CURB 65 and more than half as the scale PSI.

This study has limitations due to the fact that it is conducted at a single centre and the sample size is small, just as it is a retrospective study in which the hospital ward and ICU admission were decided at the discretion of the emergency physician, without establishing predetermined criteria. But in turn has its strengths, mainly questions the effectiveness in estimating the prognosis of the scales 65 and PSI CURB yet been recommended in the protocols of action against Influenza pneumonia $\mathrm{H} 1 \mathrm{~N} 1 \mathrm{v}$, to our knowledge is dangerous similar to primary viral pneumonia caused by bacterial agents since they have different characteristics that make both the epidemiological, clinical, laboratory parameters and clinical outcome. Based on our results, and on the understanding that this is a retrospective study with a small series of patients, it could determine that the risk indicators of adverse effects or increased mortality in pneumonia Influenza A H1N1v differ from bacterial CAP and could be summarised by the triad of refractory hypoxemia, tachypnea and bilateral radiographic infiltrates. Future well-designed studies that evaluate our observations are necessary.

We understand that the possibility of underestimating the risk and thus subjecting patients to a suboptimal treatment is high if we apply these scales in the initial management in the emergency department of primary viral pneumonia caused by influenza A H1N1v. 


\section{References}

1. American Thoracic Society Documents. Guidelines for the Management of Adults with Hospital-acquired, Ventilator-associated, and Healthcare-associated Pneumonia. Am J Respir Crit Care Med 2005; 171: 388-416.

2. Moine P, Vercken JB, Chevret S, Chastang C, Gagdos P. Severe community-acquired pneumonia. Etiology, epidemiology, and prognosis factors. Chest 1995; 107: 1182-3.

3. Fine MJ, Auble TE, Donald M. A prediction rule to identify low-risk patients with community-acquired pneumonia. NEJM 1997; 336: 243-250.

4. Lim WS, Van Der Eerden MM, Laing R, et al. Defining community acquired pneumonia severity on presentation to hospital: an international derivation and validation study. Thorax 2003; 58: 377-82.

5. IDSA/ATS Consensus guidelines on the management of CAP in adults. Clin Infect Dis 2007: 44: S27-72.

6. Pérez Padilla R, De la Rosa-Zamboni D, Ponce de León $\mathrm{S}$, et al. Pneumonia and respiratory failure from SwineOrigin Influenza A (H1N1) in Mexico. $N$ Engl J Med 2009; 361.

7. Rello J, Rodríguez A, Ibáñez P, et al; and the H1N1 SEMICYUC working group. Intensive Care adult patients with severe respiratory failure caused by influenza A (H1N1)v in Spain. Crit Care 2009; 13: R148.

8. Kumar A, Zarychanski R, Pinto R, et al. Critically ill patients with 2009 influenza A /H1N1) infection in Canada. JAMA 2009; 302: 1872-9.

9. Llorens P, Murcia J, Laghzaoui F, et al. Estudio epidemiológico de la neumonía adquirida en la comunidad diagnosticada en un servicio de urgencias: ¿influye el índice de Fine en la toma de decisiones? Emergencias 2009; 21: 247-254.
10. Niederman MS, Feldman C, Richards GA. Combining information from prognostic scoring tools for CAP: an American view on how toget the best of all worlds. Eur Respir J 2006; 27: 9-11.

11. Preparación y respuesta ante la pandemia de Gripe. Versión 11 Adaptación a la situación epidemiológica y clínica actual. Consejería de Salud Junta de Andalucía. 28 de noviembre de 2009.

12. Mulrennan S, Tempone SS, Ling IT, et al. Pandemic influenza (H1N1) 2009 pneumonia: CURB-65 score for predicting severity and nasopharyngeal sampling for diagnosis are unreliable. PLoS One 2010 Sep 21; 5: e12849.

13. Commons RJ, Denholm J. Triaging pandemic flu: pneumonia severity scores are not the answer. Int $J T u$ berc Lung Dis 2012; 16: 670-3.

14. Yandola PP, Capelastegui A, Quintana J, Diez R, Gorordo I, Bilbao A, et al. Prospective comparison of severity score for predicting clinically relevant outcomes for patients hospitalized with community acquired pneumonia. Chest 2009; 135: 1572-9.

15. Rodríguez A, Lisboa T, Rello J. Gripe A $(\mathrm{H} 1 \mathrm{~N} 1) \mathrm{v}$ pandémica en UCI: ¿qué hemos aprendido? Arch Bronconeumol 2010; 46 (Supl 2): 24-31.

16. Man SY, Lee N, Ip M, Antonio GE, et al. Prospective comparison of three predictive rules for assessing severity of community - acquired pneumonia in Hong Kong. Thorax 2007; 62: 348-53.

17. España PP, Capelastegui A, Quintana J, et al. Prospective comparison of severity score for predicting clinically relevant outcomes for patients hospitalized with community acquired pneumonia. Chest 2009; 135: 1572-9.

18. Fine MJ, Auble TE, Yealy DM, et al. A prediction rule to identify low-risk patients with community-acquired pneumonia. N Engl J Med 1997; 336: 243-50.

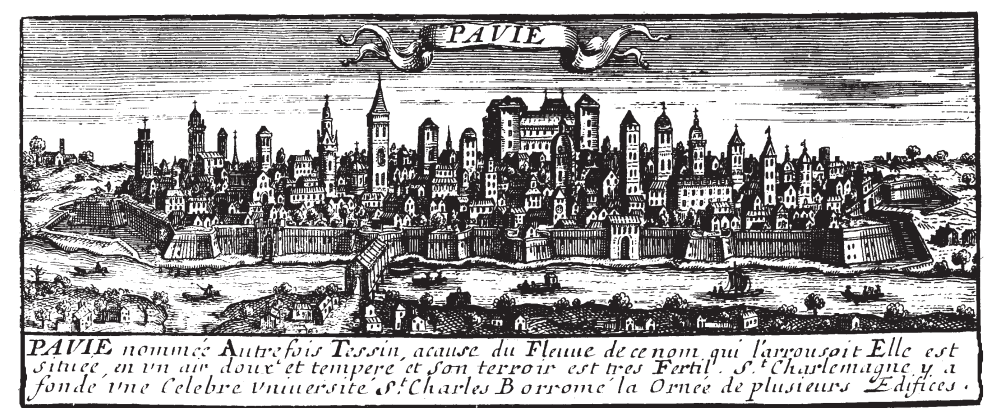

